Sylwia Szlubowska', Joanna Zalewska-Puchała', Anna Majda', Piotr Kocoń ${ }^{3}$, Jerzy Soja ${ }^{4}$, Maciej Gnass' ${ }^{2}$, Ewa Pasko², Adam Ćmiel ${ }^{5}$, Artur Szlubowski ${ }^{2}$, Jarosław Kużdżał ${ }^{3}$

'Department of Health Science, Jagiellonian University, Cracow, Poland

${ }^{2}$ Endoscopy Unit, John Paul II Hospital, Jagiellonian University, Cracow, Poland

${ }^{3}$ Department of Thoracic Surgery, John Paul II Hospital, Jagiellonian University, Cracow, Poland

${ }^{4}$ Department of Medicine, Jagiellonian University, Cracow, Poland

${ }^{5}$ Department of Applied Mathematics, AGH University of Science and Technology, Cracow, Poland

\title{
The influence of lung volume reduction with intrabronchial valves on the quality of life of patients with heterogeneous emphysema a prospective study
}

The authors declare no financial disclosure

\section{Abstract}

Introduction: A heterogeneous emphysema is one of the most severe forms of chronic obstructive pulmonary disease (COPD). In some cases, besides the standard pharmacotherapy, a new treatment option of emphysema can be used — bronchoscopic lung volume reduction (BLVR) with the use of intrabronchial valves.

Objectives: To examine the health-related quality of life ( $\mathrm{HROOL}$ ) of patients with severe emphysema after intrabronchial valve (IBV) implantation for the treatment of one lung.

Materials and methods: From 2011 to 2013 a single centre prospective observational study was performed. The study assessed the effect of the therapeutic BLVR intervention, measured by St. George Respiratory Questionnaire (SGR0). A statistical analysis by use of Wilcoxon test for dependent variables was performed.

Results: Twenty patients were enrolled to the study (mean age $63 \pm 10$ years), all ex-smokers with tobacco exposure $38 \pm 11.3$ packyears. After three months of IBV treatment the average SGRQ score improved significantly in total $(-12.8 ; p<0.001)$ and in domains and the differences were for: "symptoms" (-8.5; $p<0.001)$, "activity" $(-13.9 ; p<0.001)$ and "influence on life"(-13.5; $p<0.002)$.

Conclusions: The presented study revealed a significant improvement in the quality of life measured by SGRO after IBV treatment for heterogeneous emphysema.

For the first time our study showed the significant improvement of all three domains of SGR0 after IBV treatment.

Key words: chronic obstructive pulmonary disease, heterogeneous emphysema, quality of life, intrabronchial valve

Pneumonol Alergol Pol 2015; 83: 418-423

\section{Introduction}

Chronic obstructive pulmonary disease (COPD) is one of the most frequent chronic conditions, rated as fifth the most common cause of death and its frequency is still increasing. COPD as a progressive and irreversible disease is now one of the most essential problems of public health $[1,2]$. Evaluation of quality of life is very important for monitoring of health problems of patients suffering from COPD. Studies about quality of life are a valuable complement to the assessment of patients' clinical condition and a clue about the effects of treatment and possible improvements. For several years attempts have been made to improve to improve the quality of life of patients with heterogeneous emphysema due to COPD involving minimally invasive me-

Address for correspondence: Maciej Gnass, Endoscopy Unit, John Paul II Hospital, Jagiellonian University, Prądnicka 80, 31-202 Cracow, Poland, tel. +48 12 614 00 00,

e-mail: m.gnass@gmail.com

DOI: 10.5603/PiAP.2015.0069

Received: 11.06.2015

Copyright (C) 2015 PTChP

ISSN 0867-7077 
thods of bronchoscopic lung volume reduction (BLVR) with intrabronchial valves as an alternative to a lung volume reduction surgery (LVRS) and pharmacotherapy [3]. Previous results of treatment of severe emphysema, especially heterogenic, by LVRS seem to be more effective (reduces dyspnoea and increases lung function tests results) comparing to pharmacotherapy. However LVRS performed by thoracotomy is associated with high perioperative mortality (5\%) and higher mortality caused by pulmonary (30\%) and cardiovascular (20\%) complications mainly due to prolonged ( $>7$ days) pleural drainage caused by continued air leak [3]. The aim of the emphysematic LVRS and BLVR treatment is to eliminate the emphysematic lung tissue (usually upper lobes). BLVR methods include: intrabronchial valves (IBV, Zephyr), transbronchial stents causing so called airway bypass [4, 5], chemical blockers of bronchi such as Aeroseal, thermal vapour ablation [6] and implants, so called coils [7]. Bronchoscopic techniques may also be successfully used as a treatment of homogenous emphysema, in which surgery is not recommended [8].

The umbrella shaped valves have a framework constructed of six nitinol branches, covered with a polyurethane membrane, which causes optimal adhesion to the bronchial wall (Fig. 1) [9, 10]. These valves ensure that there is a one-way flow of air discharge from the closed part of the segment or the lobe of the lung during expiration and protect from inflow of air during inspiration (Fig. 2).

The procedure is performed during video bronchofiberoscopy under conscious intravenous sedation or by use of rigid bronchoscopy under general anaesthesia.

Current traditional methods of health assessment, which do not fulfill the holistic model of entire human well-being have been questioned, what contributed to the development of researches on patients' quality of life with the aim of making it active and simliar to the life of a healthy

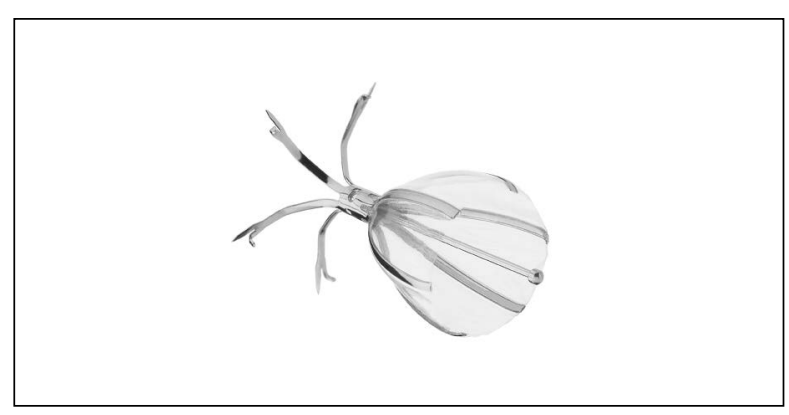

Figure 1. IBV Spiration Inc., USA; Olympus Medical Systems Corporation, Tokyo, Japan

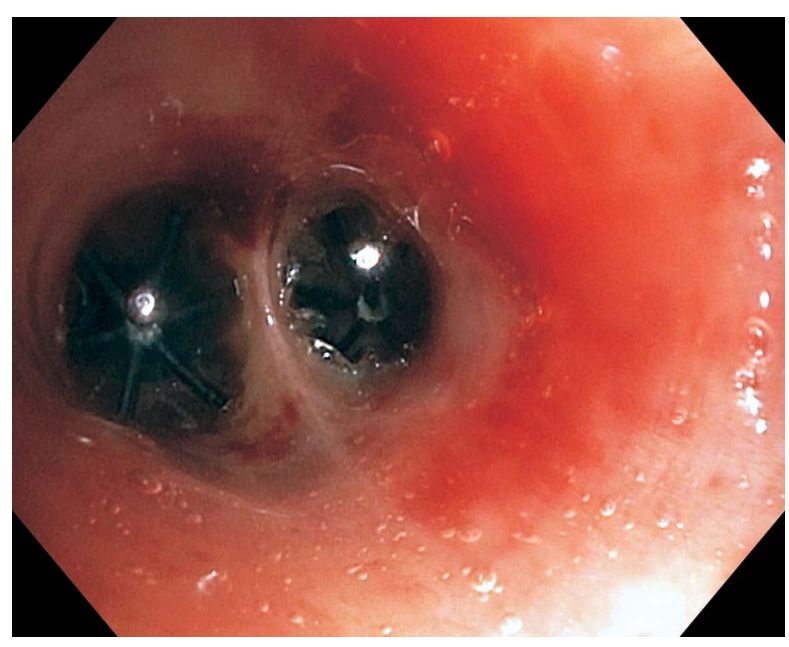

Figure 2. Two intrabronchial valves (IBV No 7) placed in the subsegmental bronchi of the right segment 3. From the Archives of Department of Thoracic Surgery and Endoscopy Unit of John Paul II Hospital, Cracow

person [11]. The assessment of quality of life during a disease, especially chronic disease is personal and its measurements may be useful for doctors and nurses in changing therapy and care [12, 13].

The aim of this study was to compare the quality of life of patients suffering from severe emphysema before and after the treatment with IBV.

\section{Materials and methods}

This prospective observational study was conducted as a diagnostic survey with an interview technique using the standardised questionnaire from the Hospital of St. George (St. George Respiratory Questionnaire - SGRQ), which was used to compare the quality of life before and after the treatment of heterogeneous emphysema with IBV. SGRQ is at present the best available tool to assess the quality of life of patients with COPD, especially when it is conducted as an interview instead of being filled in by the patient [14-15]. This questionnaire contains 50 essential questions about the quality of life of COPD patients concerning three aspects (symptoms, activity and influence on life). Every answer indicates an experimentally measured number of points, the sum of which determines a final result. The results range from 0 to 100 , where 0 means the highest and 100 - the lowest quality of life. The results of SGRQ were evaluated using a prepared spreadsheet.

Interviews with patients were conducted twice: the first time $-1-2$ days before the procedure of an implantation of IBV and the second time -3 months after the treatment with the same tool. 


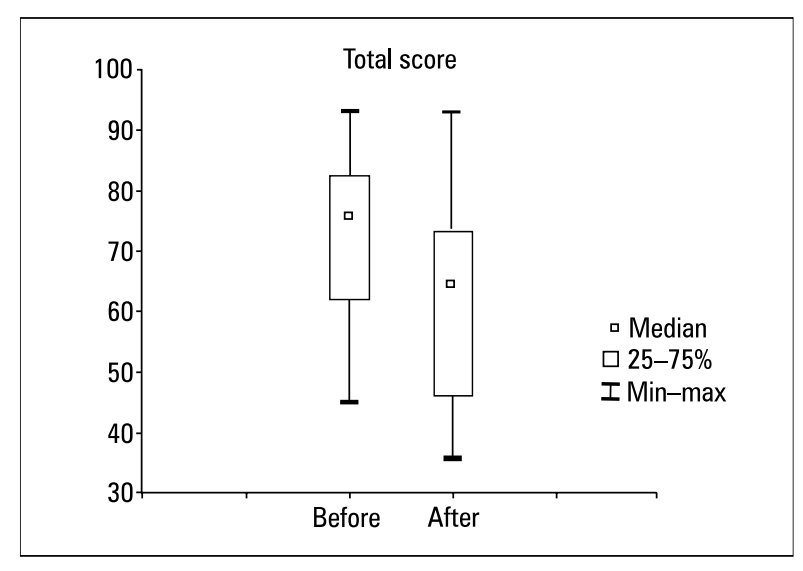

Figure 3. Comparison of a total SGRO score before and after treatment with IBV using a Wilcoxon test

Inclusion criteria for IBV implantation in our study were comparable with those used in the VENT study [16, 17]. Exclusion criteria included active infection, unstable cardiac condition, excessive sputum or the presence of giant bullae.

A statistical analysis was conducted based on descriptive statistics, i.e., the arithmetic mean with standard deviations, median, considering upper and lower quartile and minimal and maximal value. To evaluate both total SGRQ and its separate domains a Wilcoxon matched pairs test for dependent variables was used.

\section{Results}

The present study was conducted in the Department of Thoracic Surgery and Endoscopic Unit of John Paul II Hospital from October 2011 to May 2013. Due to the innovative character of the treatment of heterogeneous emphysema and the still very rare usage of implantation of IBV in Poland twenty patients suffering from heterogeneous emphysema due to COPD were enrolled to the study.

The examined group consisted of 16 men and 4 women, whose age was between 40 and 81 years, on average $63 \pm 10$ years and the duration of COPD was on average $12 \pm$ 3years. All of the patients were ex-smokers and the mean tobacco exposure was $38 \pm 11.3$ packyears and ranged between 20 and 60 packyears. Only $4(20 \%)$ of them had proven exposure to dust and chemicals. Two of them were long-standing workers of a sawmill, exposed to wood dust, oil paints and carpenter's glues. A third patient had worked in a cement works for 25 years, being exposed to a dust of cut stone and concrete, cement and asbestos. Fourth patient worked in a coal mine in direct exposure to a coal dust.
Forced expiratory volume $\left(\mathrm{FEV}_{1}\right)$ measured before treatment with IBV was $0.93 \pm 0.53 \mathrm{~L}$ and $30.87 \pm 13.62 \%$ of the predicted value, total lung capacity (TLC) was $6.21 \pm 2.43 \mathrm{~L}$ and $104.16 \pm$ $30.77 \%$ of the predicted value, residual volume (RV) was $4.42 \pm 2.15 \mathrm{~L}$ and $214.49 \pm 69.39 \%$ of the predicted value, body mass index (BMI) was $23.37 \pm 6.07$, and 6 MWT distance was 110 $\pm 111.9 \mathrm{~m}, \mathrm{PaCO}_{2}$ of less than $50 \mathrm{~mm} \mathrm{Hg}$ and a $\mathrm{PaO}_{2}$ of more than $55 \mathrm{~mm} \mathrm{Hg}$ (while breathing ambient air).

On the basis of the total SGRQ score in the examined group a significant improvement in the quality of life after treatment with IBV was observed. The mean score for this group before treatment was $73.0 \pm 15.0$, while after treatment is was $-60.2 \pm 16.6$. At least 4 points of SGRQ score improvement were obtained among $70 \%$ of patients. The mean difference between total SGRQ score before and after treatment with IBV was $-12.8 \pm 11.9 ; \mathrm{p}<0.001$. Among $25 \%$ of patients this difference reached $-28.3 \pm 11.9 ; \mathrm{p}<$ 0.0001 . The medians of scores were respectively 75.8 before and 64.4 after treatment and -10.7 for the difference (Fig. 3).

The mean score in the domain "symptoms" of the SGRQ test before treatment with IBV was $69.8 \pm 18.0$ while the mean score after treatment was $-61.3 \pm 18.6$. So after treatment a significant improvement was achieved and the mean difference of a score was $-8.5 \pm 8.5 ; \mathrm{p}<0.001$. The medians of scores were respectively 68.0 before and 59.8 after treatment and -7.7 for the difference (Fig. 4).

In this domain of the questionnaire it was observed that a cough during the three months before treatment appeared most frequently (among $40 \%$ of patients) on most of the days in a week, whereas after treatment - most commonly (also among this $40 \%$ of patients) on a few days in a week. A thick expectoration during the three months before treatment was most frequently (among 50\% of patients) present during most days in a week, while after treatment - a few days in a week. During the three months before treatment with IBV breathlessness during most days occurred among no less than $80 \%$ of patients and after treatment only among $40 \%$. After the treatment the frequency of breathlessness decreased to a few days in a week among $30 \%$ of patients and to a few days in a month among $25 \%$ of patients. Moreover it was established that during the 3 months before treatment dyspnea attacks occurred most frequently (among $40 \%$ of patients) during most of the days of a week, among $20 \%$ 


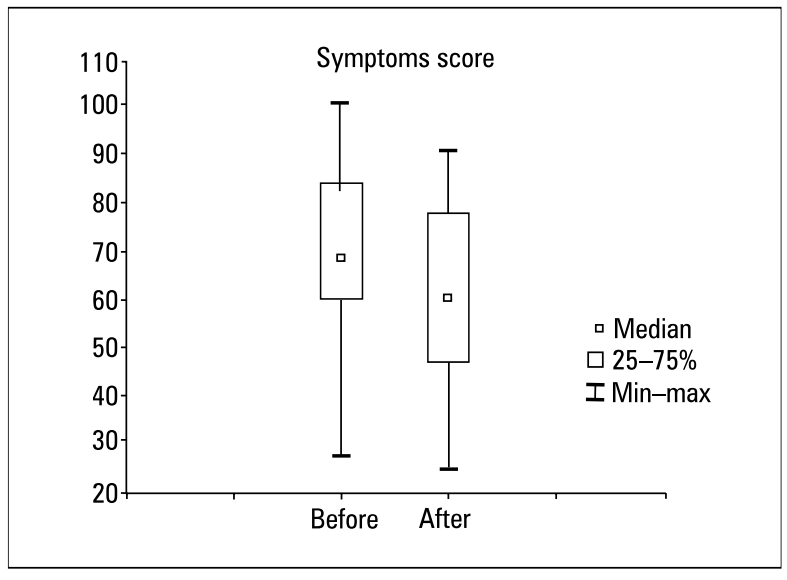

Figure 4. The comparison of a domain "symptoms" SGQR score before and after treatment with IBV using a Wilcoxon test

of patients - a few days in a week and among $20 \%$ - a few days in a month. After the treatment dyspnea attacks most often occurred during a few days of a month (35\% of patients), a few days of o week $-20 \%$, and for the most days of a week — only among $15 \%$. Before the treatment $65 \%$ of patients and after treatment only $30 \%$ of them reported respiratory symptoms during the most days of a week in last 3 months. A deterioration of symptoms usually occurred for 1-2 days regardless of performed procedure.

A mean score of the domain "activity" SGRQ before the treatment with IBV was $82.3 \pm 16.8$, whereas a mean score after treatment was $68.4 \pm$ 16.9. After the treatment a significant improvement in this domain was achieved and a difference in score was $-13.9 \pm 11.8 ; p<0.001$. The medians of scores were respectively 85.8 before and 72.3 after treatment and -12.8 for the difference (Fig. 5).

Before the treatment even a low activity such as sitting or lying (30\%), walking in a house (50\%) and on a flat terrain (60\%) caused dyspnea. After the treatment these activities caused dyspnea among a significantly lower percentage: respectively $5 \%$, $35 \%, 15 \%$ and $25 \%$. On the other hand high activity such as: going upstairs and uphill and exercise caused dyspnea among almost all patients regardless of whether they were treated with IBV or not.

A mean score in the domain "influence on life" before the treatment was $68.7 \pm 17.0$, while after the treatment $-55.2 \pm 19.0$. After the treatment a significant improvement also in this domain of SGRQ was obtained and the mean difference of the score was $-13.5 \pm 16.1 ; \mathrm{p}<0.002$. The medians of scores were respectively 73.2 before and 59.5 after treatment and -10.7 for the difference (Fig. 6).

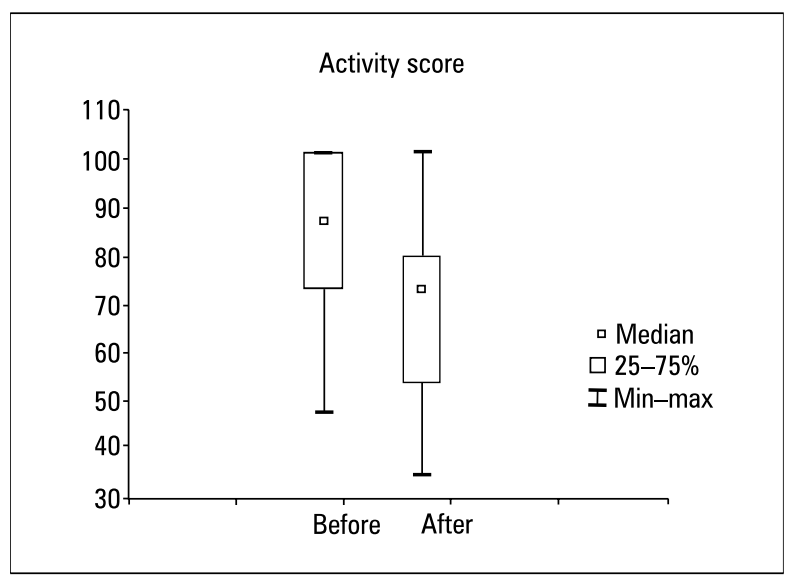

Figure 5. The comparison of a domain "activity" SGQR score before and after treatment with IBV using a Wilcoxon test

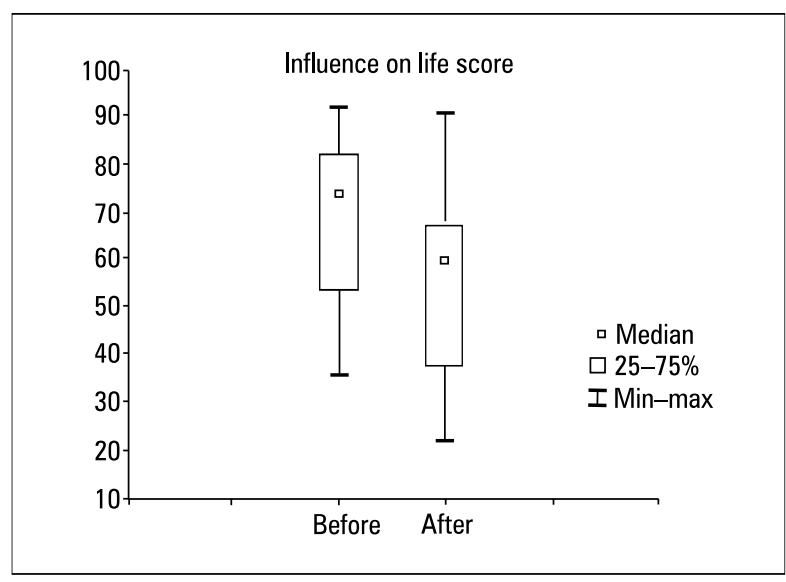

Figure 6. The comparison of a domain "influence on life" SGQR score before and after treatment with IBV using a Wilcoxon test

In this domain of questionnaire as many as $60 \%$ of patients recognized their disease as the biggest problem and reported that respiratory problems were the reason for quitting their job, regardless of the treatment with IBV. It is worth to mention that almost all examined patients were not professionally active (pension or retirement). After the treatment with IBV a percentage of patients who could not perform any wished activity due to emphysema reduced to $40 \%$. Patients declared that they experienced an improvement of free speech (without dyspnea) (by 25\%); quality of speech (by 35\%), pain during cough ( by $10 \%$ ), fatigue caused by cough (by 15\%) and general fatigue (by 10\%). After the treatment patients regarded with higher hope their health (improvement by 25\%), effects of treatment ( by $35 \%$ ) and a range of independence (shopping on their own - improvement by $20 \%$ ), they were 
not afraid to exercise (improvement by 25\%) and entertain (improvement by $20 \%$ ). Patients felt also higher self-control and more rarely got into a panic during exacerbations of the disease and overcoming obstacles required less effort (improvement by 20\%). Unfortunately, despite the treatment they still felt weak and disabled, were embarrassed in the others' presence by their symptoms, which patients regarded as bothersome for the people around them. What also did not change was a feeling of breathlessness while bending (it occurred among $75 \%$ of patients), possibility to exercise and doing all the household chores. To reassume, the quality of life of examined patients 3 months after the treatment with IBV improved significantly both when it comes to the total SGRQ score and all its domains.

\section{Discussion}

For a few years in some European pulmonological and thoracosurgical departments the minimally invasive BLVR method using intrabronchial valves has been introduced [9, 18-21]. The results of multicentre studies conducted so far are promising, although not in all of them an improvement of spirometric parameters or radiological image was not observed in all of them [9, 10, 18, 22]. However, in most of studies an improvement of the quality of life of patients treated with BLVR method was noticed [9, 16, 18, 19]. The results of a few multicentre trials using BLVR with intrabronchial valves as a treatment of severe emphysema are promising - they seem to be as effective as LVRS with significantly lower numbers of lethal and perioperative complications $[9,18]$. Nonetheless, only a few trials have shown a significant improvement of pulmonary function tests and 5-years survival after BLVR treatment [4, 21-23].

The results of a few trials indicate a significant improvement of the quality in life of severe emphysema patients treated with IBV implantation $[14,19]$. So far no studies evaluating the effects of treatment with IBV based on separate domains of the St. George Respiratory Questionnaire (SGRQ) have been conducted.

In the research of Springmeyer et al. [9] an improvement in the total SGRQ score was observed just 3 months after implantation, but a significant difference by at least 4 points was noticed among $55.7 \%$ of patients only after 6 months. In the study of Sterman et al. [18] a minimal clinically important improvement of SGRQ score (at least 4 points) was obtained after 1 month among $51 \%$ of patients, after 3 months among 53\%, after 6 months among 55\% and after a year among $57 \%$. Similarly, in our study a significant improvement of SGRQ by at least 4 points was observed after 3 months among $65 \%$ of patients. In a study of Coxon et al. [22] a statistically important correlation between an improvement of quality of life in SGRQ and a volume of the lower lobe of lung in HRCT after excluding from ventilation the upper lobe using IBV was observed. In the biggest multicentre randomized study Wood et al. [19] and Sciurba et al. [16] demonstrated a mean difference in SGRQ respectively $-10.7 \pm 11.5$ and $-11.2 \pm 14.33$ months after implantation of IBV. The results of the present study are similar to the ones quoted above, the mean difference in SGRQ was -12.8; $\mathrm{p}$ $<0.001$. In this study for the first time an attempt to evaluate 3 domains of SGRQ before and after treatment with intrabronchial valves was made. A significant improvement after the treatment in all three domains of SGRQ was proven and they amount to respectively: "symptoms" $--8.5 ; \mathrm{p}<$ 0.001, "activity" $--13.9 ; \mathrm{p}<0.001$, "influence on life" $-13.5 ; \mathrm{p}<0.002$. In the examined group the treatment with IBV significantly reduced the frequency of such symptoms as: cough, expectorating, breathlessness, and wheezes from most to just a few days of the week. After the treatment with IBV a significant improvement based on the results of the domain "activity" SGRQ was achieved only in the range of low (basic) activity, such as: calm sitting or lying, washing and dressing, walking around the house and on flat terrain. The treatment with IBV did not influence high activity, such as: going upstairs or uphill and exercising. Despite the used treatment $60 \%$ of patients regarded their disease as their biggest problem.

The study has some important limitations that must be listed. It was conducted as a single-centre study with a relatively small group of 20 patients. The follow-up period was relatively short (only 3 months), requires further observations, including 2 to 5 year survival. In this study the interlobar septum integrity was assessed by the analysis of high resolution computed tomography (HRCT) [23].The measurement of the collateral ventilation by use of Chartis Pulmonary Assessment System was not available [24].

\section{Conclusions}

The presented study revealed the significant improvement of the quality of life measured by SGRQ after the IBV treatment for heterogeneous emphysema. 
After this study the significant improvement of all domains of SGRQ: symptoms, activity and influence on life was observed for the first time.

\section{Conflict of interest}

The authors declare no conflict of interest.

\section{References:}

1. Pierzchała W, Barczyk A, Górecka D et al. Polish Respiratory Society Guidelines for Chronic Obstructive Pulmonary Disease. Pneumol Alergol Pol 2010; 78: 318-347.

2. Bąk-Drabik K, Ziora D. Quality of life in patients with chronic obstructive pulmonary disease. Pneumonol Alergol Pol 2004; 72: 128-133.

3. Van Raemdonk D, Ninane V. Lung volume reduction for severe emphysema: Do we need a scalpel or a scope? Eur Resp Rev 2010; 19: 242-247. doi: 10.1183/09059180.00005810.

4. Rendina EA, DeGiacomo T, Venuta F et al. Feasibility and safety of the airway bypass procedure for patients with emphysema. J Thorac Cardiovasc Surg 2003; 125: 1294-1299.

5. Choong CK, Macklem PT, Pierce JA et al. Airway bypass improves the mechanical properties of explanted emphysematous lungs. Am J Respir Crit Care Med 2008; 178: 902-905. doi: 10.1164/ rccm.200712-1832OC.

6. Snell GI, Hopkins P, Westall G et al. A feasibility and safety study of bronchoscopic thermal vaporablation: a novel emphysema therapy. Ann Thorac Surg 2009; 88: 1993-1998. doi: 10.1016/j. athoracsur.2009.06.038.

7. Herth FJ, Eberhard R, Gompelmann D et al. Bronchoscopic lung volume reduction with a dedicated coil: a clinical pilot study. Therap Adv Respir Dis 2010; 4: 225-231. doi: 10.1177/1753465810368553.

8. Refaely Y, Dransfield M, Kramer MR et al. Biologic lung volume reduction therapy for advanced homogeneous emphysema. Eur Respir J 2010; 36: 20-27. doi: 10.1183/09031936.00106009.

9. Springmeyer SC, Bolliger CT, Waddell TK et al. Treatment of heterogeneous emphysema using the spiration IBV valves. Thorac Surg Clin 2009; 19: 247-253. doi: 10.1016/j.thorsurg.2009.02.005.

10. Ernst A, Anantham D. Bronchoscopic Lung Volume Reduction. Pulmonary Medicine 2011; 2011. doi: 10.1155/2011/610802.

11. Tobiasz-Adamczyk B. The quality of life in social science and medicine. Sztuka Leczenia 1996; 2: 33-40.
12. Trzebiatowski J. The quality of life in perspective of social and medical science - the systematisation of definitions. Hygeia Public Health 2011; 46: 25-31.

13. Boros PW, Lubiński W. Health state and the quality of life in patients with chronic obstructive pulmonary disease in Poland: a study using the EuroQoL-5D questionnaire. Pol Arch Med Wewn 2012; 122: 73-81.

14. Kuźniar T, Patkowski J. St. George Respiratory Questionnaire as a tool to asses quality of life in respiratory system diseases. Pol Arch Med Wewn 2000; 104: 401-412.

15. Nishimura K, Tsukino M, HajiroT. Health Related Quality of Life in patients with chronic obstructive pulmonary disease. Curr Opin Pulm Med 1998; 4: 107-115.

16. Sciurba FC, Ernst A, Herth FJ et al. A randomized study of endobronchial valves for advanced emphysema. N Engl J Med 2010; 363: 1233-1244. doi: 10.1056/NEJMoa0900928.

17. Herth FJF, Noppen M, Valipour A et al. Efficacy predictors of lung volume reduction with Zephyr valves in a European cohort. Eur Respir J 2012; 39: 1334-1342. doi: 10.1183/09031936.00161611.

18. Sterman DH, Mehta AC, Wood DE et al. A multicenter pilot study of abronchial valve for the Treatment of Severe Emphysema. Respiration 2010; 79: 222-233. doi: 10.1159/000259318.

19. Wood DE, McKenna RJ, Yusen RD et al. A multicenter trial of an intrabronchial valve for treatment of severe emphysema. J Thorac Cardiovasc Surg 2007; 133: 65-73.

20. Ingenito EP, Wood DE, Utz JP. Bronchoscopic Lung Volume Reduction in Severe Emphysema. Proc Am Thorac Soc 2008; 5: 454-460. doi: 10.1513/pats.200707-085ET.

21. Venuta F, Anile M, Diso D et al. Long term follow up after bronchoscopic lung volume reduction in patients with emphysema. Eur Respir J 2012; 39: 1084-1089. doi: 10.1183/09031936.00071311.

22. Coxson HO, Nasute Fauerbach PV, Storness-Bliss C et al. Computed tomography assessment of lung volume changes after bronchial valve treatment. Eur Respir J 2008; 32: 1443-1450. doi: 10.1183/09031936.00056008.

23. Eberhardt R, Gompelmann D, Schuhmann M et al. Complete unilateral vs partial bilateral endoscopic lung volume reduction in patients with bilateral lung emphysema. Chest 2012; 142: 900-908.

24. Herth FJF, Eberhardt R, Gompelmann D et al. Radiological and clinical outcomes of using Chartis to plan endobronchial valve treatment. Eur Respir J 2013; 41: 302-308. doi: 10.1183/09031936.00015312. 